\title{
Estudio y análisis de la Contabilidad Tradicional versus Contabilidad Throughput
}

\author{
Proaño Altamirano, Gladys Elizabeth \\ Instituto Tecnológico Luis A. Martínez, Ambato, Ecuador \\ elypa6@gmail.com
}

Recibido: 23 enero 2018 Aprobado: 14 febrero 2018

\begin{abstract}
Resumen
Las empresas con el tiempo y por la aparición de mercados globalizados buscan aumentar los niveles de productividad de sus procesos. De esto nace la necesidad de crear nuevas herramientas administrativas y contables como es la Contabilidad Throughput nace de la creación de la teoría de restricciones, inventada por Dr Eliyahu Goldratt. Se presenta un nuevo enfoque para la toma de decisiones en las industrias mediante la aplicación de la teoría de restricciones (TOC). Para identificar la restricción en el sistema se realiza el análisis de los procesos de productivo a partir de su capacidad. La contabilidad de Costos fue creada pensando solo en el departamento productivo sin tomar en cuentas a las distintas áreas que existen en la empresa ya que funcionan como un cuerpo cada área necesita de la otra para poder alcanzar los objetivos organizacionales.
\end{abstract}

Palabras Claves: Metodología throughput, Toma de decisiones, Teoría de restricciones.

REVISTA DE INVESTIGACIÓN SIGMA / Vol. 05, Nº 1, 2018 (pág. 29-41) 


\title{
Study and analysis of Traditional Accounting versus Throughput Accounting
}

\begin{abstract}
Companies with time and the emergence of global markets seek to increase the enterprises over time and the emergence of global markets seek to increase the productivity levels of its processes. From this comes the need for new administrative and accounting tools such as the Accounting throughput born from the creation of the theory of constraints, invented by Dr Eliyahu Goldratt. A new approach to decision making in industry is presented by applying the Theory of Constraints (TOC). To identify the restriction analysis system processes is from productive capacity. Cost accounting was created thinking only of the production department without taking into account the different areas that exist in the company and act as a body that each area needs the other in order to achieve organizational objectives.
\end{abstract}

Keywords: Throughput, Decision Making, Theory of constraints

\section{Introducción}

En el pasado las empresas estaban preparadas y organizadas para atender a los mercados locales, lo que significaba tener una contabilidad gerencial que permitía conocer las utilidades o pérdidas, aplicando las fórmulas de costos ya conocidas, sin analizar qué es lo que más le conviene a la entidad para maximizar las utilidades (Agulera, 2008).

En la actualidad el mundo ha cambiado se ha dado paso a mercados globales donde el intercambio de los productos a nivel mundial, la competitividad, el cambio de tecnología, las empresas necesitan acoplarse a estos nuevos cambios, pues solo las que se adapten a estas nuevas tendencias de mercado logran sobrevivir, a pesar de esto la contabilidad gerencial no ha cambiado (Suarez, 2003).
Además, en estos tiempos han aparecido muchas herramientas administrativas orientadas a solucionar problemas fácilmente, expandir las empresas como es kaizen, mejora continua, six sigma, técnica Smed, estrategia de localización buscando de esta manera responder a las nuevas exigencias de los clientes, siendo más flexibles para satisfacer las necesidades del consumidor (Ortiz, 2013).

Es importante señalar que las ciencias contables aporta al nuevo conocimiento y a su vez genera herramientas que permiten un uso adecuado de sus compuestos como los costos producción y comercialización sin dejar de lado su entorno interno y externo.

Finalmente, los gerentes están buscando una manera que la contabilidad se adapte también a estos nuevas exigencias del mundo 
globalizado (tecnología, competitividad, nuevos mercados, productos, exigencias del consumidor) apareciendo la metodología de contabilización denominada Contabilidad Throughput, (metodología de las restricciones) que fue el pensamiento de Eliyau Goldratt su creador, la misma que es más fácil, entendible y ayudar a tomar decisiones gerenciales maximizando las utilidades y ayudando a responder a las exigencias del cliente actual (Caparros, 2001).

\section{Desarrollo}

Desde inicios del siglo pasado, la sociedad y las organizaciones han cambiado enormemente, y el entorno de los negocios continúa cambiando cada vez más (Corbett, 2004).

"ElDrEliyahu Goldratt busca la necesidad de cambiar la gerencia, de ver la empresa como un sistema, de la necesidad de convertir la empresa en una organización que aprende, pero nos ofrece las herramientas que nos puede permitir lograr esa revolución. Estas herramientas son los Procesos de Pensamientos de Goldratt. Esta es la base para crear la organización que causa el cambio, y en consecuencia, una que ingresa a un paso de mejora continua" (Ferro, Pedraza, $\&$ Hernández, 2011).

Actualmente la participación de la mano de obra directa sobre el total de los costos de las empresas se ha reducido, en muchos casos no es mayor del $10 \%$. Aun así la mayoría de empresas continúan utilizándola como la base de asignación de los Gastos de Operación.

Muchos consideran que esta es la causa de que la información de la contabilidad es errada, y el verdadero objetivo de la contabilidad gerencial se ha olvidado y la información es distorsionada $\mathrm{y}$ en consecuencia se toma decisiones erradas llevando esto a la liquidación de muchas empresas (Marin \& Gutiérrez, 2013).

Además, la obsolescencia de la contabilidad de costos no se debe a que solamente utiliza una actividad como medidor para asignar costos, sino debido a que asigna costos a los productos. La obsolescencia de la asignación proviene del hecho que los gastos que son asignados, bajo cualquier sistema de asignación, no varían directamente con el volumen de producción y/o la mezcla, o de acuerdo con ninguna otra variable. Por tanto, la asignación solamente nos confunde y nos lleva a tomar decisiones irracionales (Suarez, 2003). 


\section{Tabla 1}

Comparación de la Contabilidad de Costos y la Contabilidad Throughput.

\begin{tabular}{ll} 
Contabilidad de Costos & Contabilidad Throughput \\
\hline Muy complicada & Simple \\
\hline Muy pocos la comprenden & Se entiende fácilmente \\
\hline $\begin{array}{l}\text { No permite identificar los productos que más } \\
\text { contribuyen a las utilidades de la empresa }\end{array}$ & $\begin{array}{l}\text { Identifica los productos que más contribuyen } \\
\text { con la idea de utilidades }\end{array}$ \\
\hline No ayuda a las empresas a prosperar & $\begin{array}{l}\text { Ayuda a los gerentes a tomar decisiones } \\
\text { rápidamente. }\end{array}$ \\
\hline
\end{tabular}

Reducir costos y gastos para lograr utilidades Incrementar la velocidad que el dinero ingresa a la empresa mediante las ventas

Puede ser directos o indirectos

Solo se consideran los variables

Fuente: (Corbett, 2004) - (Zambrano Saltos \& Castillo Baque, 2017)

\section{Terminología}

\section{ABC-Costeo basado en actividades:}

Este sistema de Contabilidad Gerencial de Costos permite la asignación y distribución de los diferentes costos indirectos de acuerdo a las actividades realizadas, identificando el origen del costo de la actividad, no sólo para la producción sino también para otras áreas como distribución $\mathrm{y}$ ventas.

CDP-Capacidad disponible de planta: Es la capacidad, en minutos, que tiene la planta para q producir. Es igual a la suma de capacidad de todas las maquinas.

CTV - Costo totalmente variable: Se define como el costo que varía directamente con el volumen de producción. Si la empresa produce y vende una unidad delproducto.va a incurrir en este costo, y si no produce ninguna unidad no incurrirá en este costo.

MP: Materia Prima.

TOC: Teoría de Restricciones.

TTPP -Tiempo total de proceso por producto (TTPP): Es el tiempo que cada producto usa de la Capacidad Disponible de la Planta (CDP, en unidades de tiempo).

\section{RRC - Recursos con restricción de} capacidad (Caparros, 2001).

\section{Historia de la contabilidad trúput}

Según Corbett (2004), dice que TOC se inició en los 70s, cuando el físico israelí Eliyahu Goldratt comenzó a estudiar los problemas de la logística de producción. Él no tenían ningún antecedente en negocios, pero utilizó los métodos de solución de problemas que aprendió en física 
para buscar resolver los problemas de la logística de producción.

Goldratt creó un método completamente nuevo para la logística de producción, aunque no tenía conocimientos previos de los métodos existentes. Estaba intrigado por el hecho de que las metodologías de producción tradicional no tenían ningún sentido común. Su método fue muy exitoso, y muchas empresas se interesaron. Entonces Goldratt se dedicó a desarrollarlo.

A comienzos de los 80 s escribió un libro acerca de su teoría. El libro, La Meta, fue escrito en forma de novela y mostraba las dificultades de la lucha de un gerente de planta para manejar el negocio. A medida que la historia avanza, el gerente comienza a descubrir los principios de la teoría de Goldratt, y la empresa recupera su competitividad. El éxito del libro fue, y aún lo es, enorme. Muchos gerentes han leído el libro y comienzan a aplicar los principios de TOC.

En el libro, Goldratt critica los métodos tradicionales de la gerencia, incluyendo a la contabilidad de costos.

Muchas empresas que estaban aplicando la logística de producción de Goldratt incrementaron el desempeño de su producción hasta tal punto que los problemas comenzaron a surgir en otras áreas, como en la logística de distribución y la gerencia de proyectos.

\section{Metodología throughput}

El TOC se basa en el principio de que existe una causa común para muchos efectos, y que los efectos que vemos y sentimos son una consecuencia de caudas más profundas, este principio nos conduce a una visión sistemática de la empresa. (Pérez \& Arcos, 2009)

La restricción de esta teoría podemos expresar en palabras más cortas y entendibles que son: cualquier cosa que limita un sistema de alcanzar un mayor desempeño en relación con su meta (González, Mosquera, \& Cadavid, 2003).

\section{Proceso de la contabilidad throughput}

Según (Duque, Velásquez, \& Cadavid, 2004) el proceso de la contabilidad throughput se basa en:

\section{Identifique las restricciones del sistema}

En una organización siempre existirá un recurso límite de su máximo flujo, para aplicar este método debemos identificar al eslabón más débil, el flujo máximo se lo denomina ${ }^{1} \mathrm{RRC}$. Existen limitaciones internas, tales como las 
limitaciones de capacidad del equipo productivo, la plantilla, etc. Y otras limitaciones externas que vienen impuestas por el mercado tales como plazos de entrega, diseño.

\section{Decida como explotar las restricciones del sistema}

Una vez identificado el motivo por el cual se reduce el rendimiento del recurso, necesitamos obtener el máximo de él, necesitamos garantizar que siempre exista un amortiguador. Es decir, sacar el máximo provecho de las limitaciones, gestionándolas de forma eficaz.

\section{Subordinar todo lo demás a la decisión anterior}

Todos lo demás recursos deben trabajar al mismo tiempo de la restricción ni más rápido ni más despacio. La restricción no puede detenerse porque eso significaría que todo pare (Pérez \& Arcos, 2009).

\section{Elevar la restricción del sistema}

En este paso utilizamos obtener al máximo la restricción, considerando varias alternativas para invertir la restricción. Consiste en aumentar la capacidad de la limitación, aplicando un programa de mejora del nivel de actividad de la restricción. Hay que evitar que el recurso limitado pierda tiempo o realice actividades que pueden hacer otros recursos.

\section{Si en un paso previo se ha roto una restricción, vuelva al paso 1}

Cuando se rompe o se supera la limitación, generalmente, no se retrocede para volver a examinar las reglas y se tiende a caer en la inercia, se ha creado entonces una nueva limitación política. Es preciso trabajar en forma permanente con las nuevas limitaciones que se presenten para que no se produzca inercia (Caparros, 2001).

"Pero No Permita que la Inercia Genere una Restricción en el Sistema” (Corbett, 2004) 
Tabla 2

Tipos de Restricciones

TIPOS DE RESTRICCIONES CONCEPTO

Restricción de Manufactura $\quad$ Esta restricción se refiere los obstáculos que tenga en la capacidad de producir.

Restricción de Mercados

Existe cuando la demanda del mercado es menor que la capacidad de producción de la empresa.

Restricción de Materiales

Existe cuando la calidad, cantidad, y oportunidad de los materiales nos impide cumplir con la demanda.

Restricción de Logística

Problemas en los métodos de trabajo, administración, adquisición de materia prima.

Restricción de Políticas

Son políticas, leyes implantadas por los gobiernos que obstaculizan el desempeño productivo.

Fuente: (González, Mosquera, \& Cadavid, 2003)

\section{La contabilidad del throughput los cuales pueden ser:}

\section{Fundamentos}

Para el creador de la teoría de la restricciones Goldratt, la contabilidad tradicional está obsesionada por el «pensamiento del mundo del costo» que puede conducir a una espiral declinante de la reducción del coste (Ortiz, 2013).

La Contabilidad del Throughput fue creada única y exclusivamente para expresar los extremos relativos a la Teoría de las Limitaciones en términos monetarios (Caparros, 2001).

El objetivo de una empresa es ganar dinero, según Chango (2015), existe una curva de aprendizaje que permite obtener indicadores para saber si una empresa está ganando dinero,

\section{El beneficio neto.}

\section{El Rendimiento sobre el capital invertido} (ROI).

3. La liquidez o flujo de caja (Marin \& Gutiérrez, 2013).

Los indicadores financieros no encaja del todo con la Teoría de las Limitaciones, pues, ésta fue concebida y desarrollada en relación a empresas de producción, transformación pero solo basándose en el departamento de producción de una gran empresa ignorándose totalmente en su análisis la existencia de otras áreas (Corbett, 2004). 
La adaptación de los indicadores $\quad \mathbf{P}=$ Precio unitario por producto.

financieros adoptando la metodología throughput

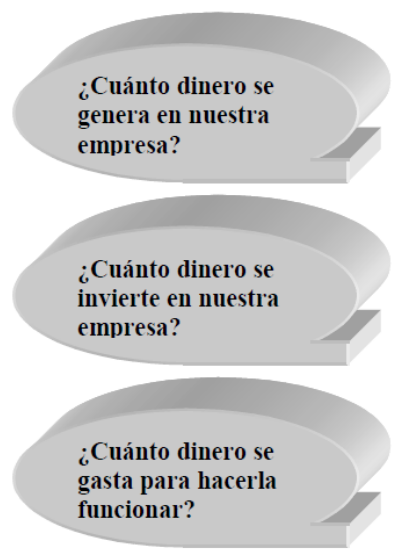

Figura 1. Medidas Operativas (Suarez, 2003)

\section{Trúput unitario}

Según Corbett (2005) manifiesta que que trúput es todo dinero que ingresa a la empresa menos el dinero que se cancela a los proveedores.

\section{Fórmula:}

$\mathbf{T u}=\mathrm{P}-\mathrm{CVT}$ donde:

$\mathbf{T u}=$ Trúput por unidad de producto.
$\mathbf{C V T}=$ Costos totalmente variables

\section{Trúput total}

Mediante esta fórmula se obtendrá la tasa de generación de todos los productos que la empresa necesite analizar aplicando la fórmula de trúput total (Zambrano Saltos \& Castillo Baque, 2017).

$\mathbf{T T P}=(\mathrm{Tu})(\mathrm{Q})$ donde:

$\mathbf{T T P}=$ Trúput total por producto.

$\mathbf{T u}=$ Trúput por unidad

$\mathbf{Q}=$ Cantidad vendida en un período.

Analizaremos el ejemplo de la empresa $\mathrm{XYZ}$ que tiene dos productos: producto $\mathrm{X}$ y el producto Y con los siguientes datos:

\section{Tabla 3}

\section{Cálculo de Costeo Trúput}

\begin{tabular}{lcc} 
Variables & Producto X & Producto Y \\
\hline Precio del Producto P & 200 & 250 \\
\hline Costos Totalmente Variables & 80 & 100 \\
CTV & & \\
\hline Cantidad Vendida Q & 180 & 200 \\
\hline Cálculo del Trúput & & 150 \\
\hline Tu $=$ P - CTV & 120 & 30.000
\end{tabular}

Fuente: Elaboración Propia 
Como se muestra en la tabla el cálculo del Trúput de los productos X y Y, para obtener el cálculo de costo unitario Trúput mediante la fórmula, el precio de producto menos el costo total variable así mismo el Trúput total es el resultado de multiplicar ( $\mathrm{P}^{*} \mathrm{Q}$ ) (Zambrano Saltos \& Castillo Baque, 2017) .

\section{Indicadores de operación trúput}

1. Throughput (T): es la tasa a la que el sistema genera dinero por medio de las ventas. Es el dinero fresco que entra a la compañía. El throughput es el único indicador que está directamente identificado con el producto y tiene dos aspectos fundamentales: el ingreso y los costos totalmente variables. (Pérez \& Arcos, 2009)

2. Inventario (I): es todo el dinero que el sistema invierte en comprar cosas que luego se tiene la intención de vender. Se define como el dinero que está todavía en el sistema. (Pérez \& Arcos, 2009)

3. Gastos de operación (GO): Es todo el dinero que el sistema gasta convirtiendo la inversión en throughput A diferencia de la contabilidad de costos tradicional, en este concepto se incluye tanto el costo de la mano de obra directa como los gastos de fabricación, de venta y administración. Todos ellos son tratados como gastos de período. Los indicadores financieros o globales se construyen con los indicadores operativos de la siguiente manera: (Zambrano Saltos \& Castillo Baque, 2017)

Utilidad Neta $(\mathbf{U N})=$ throughput $(\mathrm{T})-$ gastos de operación $(\mathrm{GO})$

\section{Retorno sobre la inversión $($ ROI $)=($ throughput} (T) - gastos de operación (GO))/ Inventario (I)

Una vez Identificadas las capacidades y con base en el cuadro de maquinaria paralos tres procesos en estudio, no se encuentran restricciones en cuanto la maquinaria y se determina la posibilidad de cumplir con las demandasen los tres procesos. Si se analizan los estados de resultados de la compañía con la metodología tradicional de costos, se observa que los costos de la mercancía vendida (CMV) incluyen los costos de materias primas o productos químicos para los procesos de lavandería los costos de mano directa y los costos indirectos de fabricación( CIF) en forma global. Esta metodología oculta el procedimiento efectivo para establecer si la producción de un producto analizado a partir de sus costos totalmente variables puede ser rentable o no, puesto que se le cargan al producto los sobre costos causados por agentes externos (Pérez \& Arcos, 2009). 
En la contabilidad del throughput se consideran únicamente los costos de la mercancía vendida (CMV) y los costos totalmente variables (CTV) para calcular el throughput, indicador que define la rentabilidad del producto o proceso y con el cual es posible establecer las estrategias del negocio (Pérez \& Arcos, 2009).

\section{Throughput =VENTAS - CTV}

CTV $=$ costos directos de fabricación (incluye las materias primas y el agua utilizada en los procesos).

Al realizar este análisis para cada uno de los productos que la compañía produce, podemos observar cuál es el producto más rentable, el que más dinero produce y esto es lo que se denomina throughput. (Pérez \& Arcos, 2009)

\section{Resultados}

Los resultados obtenidos de esta investigación no es aplicable para todas las empresas se deberá realizar un análisis previo junto con la gerencia, para conocer si la implementación de este tipo de contabilidad es recomendable.

En períodos y sectores donde la competencia se intensifica y solamente permanecen en el juego los más adaptados, la necesidad de mejorar se convierte en condición necesaria para la supervivencia y, ahora sí, se intentan adoptar las prácticas de dirección de los ganadores. Por ello, a veces se exaltan e incluso se exageran, por parte de algunos autores, las virtudes de las prácticas empresariales de dirección más exitosas y no se es lo suficientemente consciente de que se tratan de actuaciones no fáciles de imitar, ni están exentas de riesgos. Lo que antes que nada habría que plantearse es si la filosofía que subyace en la Teoría de las Limitaciones, es solamente aplicable a una parte de la empresa y debe ser considerada como una técnica aplicable al ámbito de la producción, o si por contra, bajo estas prácticas se encuentran una serie de principios de validez general aplicables a la empresa en su conjunto e incompatible con los fundamentos teóricos de nuestra disciplina. Esta es la cuestión principal. Lo que está claro es las múltiples contradicciones, por llamarlo de alguna manera, con las que nos hemos encontrado en las propuestas de Goldratt, con respecto a la contabilidad de gestión. Con una importante dosis de buena voluntad, por nuestra parte,

Podría pensarse que estamos únicamente ante un problema de interpretación metodológica y terminológica simplemente, lo que creemos que en modo alguno sea cierto, pues pensamos 
que ha quedado claro que utiliza los parámetros de acuerdo con su conveniencia.

Consecuentemente, desde esta perspectiva y en consonancia con el tratamiento que hemos realizado en este trabajo, la teoría de las Limitaciones y la Contabilidad del Throughput se deben considerar, como ya se ha planteado aquí, como un modelo quiere que hagamos las cosas de un modo algo distinto, pero no que reconfiguremos nuestras pautas fundamentales de pensamiento sobre la Contabilidad de Gestión, ni que hagamos trizas el orden establecido, como algunos pretenden.

\section{Conclusiones}

La aplicación de herramientas administrativas ha permitido que la Contabilidad también evoluciones y cambie buscando acoplarse al mundo actual ya que la contabilidad

\section{Tabla 4}

Costos por Absorción Versus Trúput

CONTABILIDAD DE COSTOS POR ABSORCIÓN de costos fue realizada en base al departamento de producción sin considerar todos los departamentos de la empresa (Ortiz, 2013).

Estas técnicas evitan la adopción de prácticas inadecuadas en el manejo de las operaciones de una compañía, razón por la cual muchas de estas organizaciones ven reducido su nivel de utilidades dada la disminución de los ingresos y/o aumento de los costos operacionales correspondientes.

La lógica y metodología de priorización de contabilidad del throughput es irrefutable, sin embargo su aprovechamiento y funcionalidad se ven limitadas por el tipo de empresas o los diferentes tipos de productos que se elaboren en donde se busque aplicar esta teoría (Caparros, 2001).

CONTABILIDAD TRUPUT

SORCION

\begin{tabular}{ll}
\hline Prioriza por Margen & Prioriza por Tiempos en el RRC \\
\hline Margen= Precio-(CD-(\%Asignac) x Indirectos)) & Trúput=Precio- CTV \\
\hline Elimina los menos rentables que superen capacidad & $\begin{array}{l}\text { Elimina los que generen menos Trúput por tiempos en } \\
\text { el RCC }\end{array}$
\end{tabular}

Fuente: Elaboración Propia

REVISTA DE INVESTIGACIÓN SIGMA / Vol. 05, Nº 1, 2018 (pág. 29-41) 
Para concluir es importante señalar la importancia que ha tomado esta denominada contabilidad TRUPUT en el desarrollo local de la localidad por lo tanto señalamos su aplicación en la empresa Plasticaucho Industrial S.A., en ciertos procesos como:

- Manejo de Inventarios.

- Control de Amortiguadores.

- Aprovisionamiento con proveedores.

En Plasticaucho Industrial S.A. no se aplicó en todas las raíces inclusive en nota de pedidos y la misma contabilidad, ya que se tenía que llevar otra contabilidad para fines Tributarios lo que provocaba más recursos en tiempo, material y personal.

Se pude concluir que la Metodología Throughput es una Contabilidad Gerencial que ayuda a la toma de decisiones gerenciales, su aplicación va a depender del tipo de empresa que se aplica, los recursos como: personal, materiales, áreas entre otras.

\section{Referencias bibliográficas}

Agulera, C. (2008). Un Enfoque Gerencial de la Teoria de las Restricciones. Revista de Administración de Empresas - Estudios Gerenciales, 3-8.

Caparros, M.(2001). La Teoría de las Limitaciones y la Contabilidad del Throughput, Un Paradigma de Finales de Siglo XX. Especial Encuentros , 9-16.

Corbett, T. (2004). Contabilidad del Trúput. Estados Unidos: North River Press.

Chango, M. (2015). El conocimiento y la experiencia potencian la productividad. ESPEActum, 1(2), 17-25.

Duque, D.; Velásquez, P. \& Cadavid, L. (2004). Hacia una Nueva Métrica Financiera Basada en Teoría de Restricciones. Estudios Gerenciales, 3-10.

Ferro, R.; Pedraza, L. \& Hernández, C. (2011). Maximización del Throughput en una Red de Radio Cognitiva Basado en la Probabilidad y Falsa Alarma. Tecnura, 3.

González, J.; Mosquera, K. O. \& Cadavid, L. (2003). Desarrollo de una Metodologia de Implementación de los Conceptos de Toc para Empresas Colombianas. Estudios Gerencias, 1-23.

Marin, W. \& Gutiérrez, E. (2013). Desarrollo e Implementación de un Modelo de Teoría de Restricciones para Sincronizar las Operaciones en la Cadena de Suministro . Revista EIA, 667-77.

Ortiz, M. (2013). Teoría de Restricciones y Modelación PL como Herramientas de Decisión Estratégica para el Incremento de la Productividad en la Línea de Toallas de una Companía del Sector Textil y de Confecciones . Prospect.Vol.11., 21-29.

Pérez, C. \& Arcos, L. (2009). Toma de Decisiones 
Rentables Mediante la Contabilidad del

Trúput en una Lavandería Industrial.

Revista Soluciones de Postgrado EIA, 229-241.

Suarez, M. (2003). Análisis Comparativo Sobre la Aplicación de la Contabilidad del Trúput frente a la Contabilidad de Costos Tradicional . Finanzas y Negocios Tradicionales, 23-24.

Zambrano Saltos, T. \& Castillo Baque, D. (2017). La Contabilidad Del Trúput Y Su Influencia En El Mejoramiento De Los Resultados De Las Empresas. Revista Observatorio de la Economía Latinoamericana. 